\title{
Relaciones de la facultad de medicina con el hospital universitario. La enseñanza de la medicina clínica
}

\author{
J. Millán Núñez-Cortés, J. García-Seoane, E. Calvo-Manuel, R. Díez-Lobato, F. Calvo-Manuel, \\ A. Nogales-Espert
}

Las nuevas necesidades en la formación de los futuros médicos, particularmente aquéllas relacionadas con la definición, adquisición y evaluación de competencias, necesitan una potente estructura hospitalaria que soporte la docencia práctica en habilidades clínicas. El trascendente papel reservado a los hospitales universitarios significa que la institución sanitaria debe adaptarse para facilitar la implantación de una enseñanza de calidad, en aspectos estructurales y en el proceso de definición de objetivos y de evaluación de resultados a través de pruebas objetivas. Ello implica cambios en la estructura, en la organización y en la gestión.

Relationships between the Faculty of Medicine and the University Hospital. Teaching clinical medicine

The training of future doctors entails a series of new needs, especially in relation to the definition, acquisition and evaluation of abilities, and this requires the backing of a powerful hospital structure that is capable of offering the practical teaching and learning of clinical skills. The fundamental role played by university hospitals means that the health care institution must adapt itself to facilitate the implementation of high quality teaching, both as regards structural aspects and in the process of defining aims and evaluating results by means of objective testing. This involves changes in the structure, the organisation and management.

\section{Introducción}

Para satisfacer las demandas formativas del futuro médico, el hospital universitario se constituye como un elemento trascendental. Una educación médica basada en la adquisición de competencias profesionales específicas, entre las que las habilidades y las destrezas en procedimientos diagnósticos y/o terapéuticos son protagonistas, no se puede desarrollar fuera del ambiente hospitalario. Al mismo tiempo, e independientemente de que numerosas actividades formativas en este sentido deban sustentarse en la utilización de los recursos asistenciales de una forma programada y previamente planificada, forma parte de la adquisición de los valores clínicos y profesionales la incorporación y el trabajo del alumno incorporado a las unidades clínicas, donde es capaz de vivir la realidad de la medicina como ciencia, del sistema sanitario, y de la utilización de los recursos asistenciales de una manera correcta. De esta forma, la formación en el seno del hospital se configura como un equilibrio entre actividades predefinidas y otras que se realizan de manera 'oportunista' en función de la vida diaria de la asistencia a los enfermos y de todo lo que eso lleva consigo (asistencia a sesiones, interconsultas, ampliación de informes, situaciones de urgencia sobrevenida, etc.).

Finalmente, el alumno debería alcanzar un conjunto de saberes por una parte, de habilidades por otra, y de actitudes, también, que le permitan conocer más y mejor lo que debe hacer en el ejercicio profesional, cómo debe hacerlo, y le afirmen la convicción del papel que ocupa el médico en la asistencia a los enfermos, a través de la adquisición paulatina de valores profesionales que no siempre son tangibles.

De esta forma, la asistencia a las actividades clinicoasistenciales debe ser algo más que los simples comentarios de historias clínicas, de evolutivos o de exploraciones complementarias, que es, desafortunadamente, en lo que se con-
Facultad de Medicina. Universidad Complutense. Madrid. España.

E-mail jesus.millan@salud.madrid. org 
Tabla I. El hospital en la adquisición de competencias clínicas.

\section{Estructura}

- Mayor implicación de la organización sanitaria en la docencia práctica

- Nuevo modelo de relación de los profesionales sanitarios con la universidad

- Reconocimiento e incentivación de las actividades docentes

- Cartera de servicios de las unidades asistenciales

Proceso

- Establecimiento de objetivos

- Diseño del itinerario formativo

- Evaluación del proceso formativo y retroinformación

Resultados

- Planes de estudio basados en competencias clínicas

- Participación de expertos en educación médica

- Evaluación objetiva de las competencias adquiridas

vierten un porcentaje considerable de las actividades prácticas de nuestros alumnos. Si no somos capaces de predefinir e incluir en los programas e itinerarios formativos determinadas prácticas de habilidades o procedimientos será difícil conseguir que el alumno adquiera competencias propias para poderlas poner en práctica de manera autónoma o tutelada, según el caso. De esta forma, sin menoscabo de la utilidad del aula, del seminario, del laboratorio o del taller, son las salas de hospitalización, las consultas, los bloques quirúrgicos, las áreas de urgencias o críticos, o las consultas de primaria, las que van a permitir al alumno adquirir ciertas capacitaciones para actuar de una forma independiente y con alguna o suficiente experiencia.

\section{El hospital en la adquisición de competencias clínicas}

En este sentido, nos parece clave señalar la importancia del hospital en la adquisición de las competencias clínicas, si es que el hospital pretende -firmemente y con razón- denominarse universitario.
Tabla II. Algunos indicadores hospitalarios para las unidades asistenciales.

Indicadores de investigación

- Publicaciones/año

- Factor impacto tota

- Comunicaciones científicas/año

- Ensayos clínicos/año (activos)

- Proyectos de investigación/año (activos)

- Tesis doctorales presentadas/leídas

- Tesis doctorales/año (en desarrollo)

- Porcentaje de médicos internos residentes con tesis doctoral en realización/terminada

- Porcentaje de personal con título de doctor

Indicadores de docencia

- Sesiones clínicas/mes

- Otras sesiones/mes

- Número de médicos en formación

- Propia especialidad

- Distinta especialidad

- Becarios/estancias

- Número de profesores

- Funcionarios

- Contratados

- Colaboradores

- Asignaturas con carga docente y número de créditos (todos los niveles de docencia: grado, posgrado, licenciaturas, diplomaturas, títulos propios...)

- Carga lectiva práctica por profesor y año (número de alumnos $\times$ horas de estancia/número de profesores)

- Cursos/seminarios organizados/impartidos

- Actividades de formación continuada acreditadas (Sistema Nacional de Salud)

- Número de créditos reconocidos, ofertados para formación continuada

Y lejos de participar de una forma indeterminada y ambigua, los hospitales universitarios deberían adaptar algunas de sus características e incorporar consideraciones específicas que ayuden a cumplir la función -por otra parte, tan noble- de formar profesionales para el ejercicio posterior de las profesiones sanitarias. Y esto debe de afectar a todos los elementos claves de la enseñanza: la estructura del centro de enseñanza, el proceso educativo en sí mismo y los resultados educativos (Tabla I).

El hospital universitario precisa incorporar cambios estructurales y funcionales que permitan una mayor y mejor implicación de la organización sanitaria a la docencia de la medicina. Se precisan cambios en la propia organización, en la 
Tabla III. Enseñanza de las competencias clínicas en el hospital universitario.

Definición de las competencias clínicas

- Catálogo de habilidades y competencias clínicas

- Cronograma de adquisición

- Ámbito de aprendizaje (aula, seminario, laboratorio, hospitalización, consultas, urgencias, centros de salud, laboratorio de habilidades...)

- Método de aprendizaje (casos-problema, pacientes reales, pacientes estandarizados, maniquíes, talleres, laboratorio...)

Metodología educativa

- Integración de conocimientos

- Resolución de problemas

- Adquisición de competencias clínicas

- Nuevo diseño de recursos educativos

- Nuevas técnicas de evaluación

- Campo virtual para la enseñanza

Pruebas de evaluación

- Necesidad inherente al proceso formativo

- Alto valor en la formación (alumno, profesor, institución)

- Enfocadas a los objetivos docentes

- Basada en evidencias

- Basada en estándares: requisitos, niveles, contexto

- Con cualidades intrínsecas: válida, fiable, aplicable, justa, reproducible

'cultura' que reina en ella y en la gestión del propio centro, orientándola hacia valores propios de la docencia y de la investigación, y no sólo de la asistencia (Tabla II).

Se precisa establecer un nuevo modelo de relación de los profesionales sanitarios del hospital con la universidad. Es preciso, asimismo, un mayor y más cualificado reconocimiento e incentivación de las actividades docentes. Y, finalmente -por sólo citar los elementos básicos-, se necesita una permanente armonización entre las unidades docentes con la estructura asistencial y la cartera de servicios de cada centro, condición para la que es determinante la figura y la responsabilidad del profesor de medicina clínica con plaza asistencial vinculada. Resulta obligado mejorar la gestión docente, a través de la coordinación de personas y funciones con responsabilidad docente y asistencial, y fomentar la relación interdepartamental y la creación de unidades propias de gestión de la docencia. Quizás un elemento crítico en la mejora de la estructura docente hospitalaria es la implicación del médico en la enseñanza de la medicina. Sólo a través de una mejora en las capacidades docentes del médico, en el reconocimiento e incentivación de dicha labor, y en la formación del médico como 'docente' (mayor profesionalización de la docencia) podremos convertir el hospital en un lugar en el que se asuma, de forma insensible, que entre sus misiones se encuentra la de enseñar medicina.

Pero es preciso ir más allá, y detallar objetivos señalando cómo, dónde y cuándo se adquieren las competencias, y de qué forma se evaluarán. El hospital ha de ser el lugar donde el alumno pueda realizar un itinerario formativo completo de acuerdo con los objetivos educativos marcados; en definitiva, donde pueda adquirir y ser evaluado de la faceta clínica. Por lo tanto, será necesario que, además de los aspectos de estructura hospitalaria, se cuiden aquéllos concretos sobre el proceso educativo: definición de objetivos/contenidos (conocimientos, habilidades, actitudes) y programación de actividades (teóricas, prácticas y de inspiración de actitudes) que permitan su consecución, estableciendo la forma de control y de evaluación (tan limitada en este momento). En este sentido, y a nuestro juicio, resulta prioritario el fomento de la figura del tutor de formación, persona de la plantilla del hospital que conoce y controla el proceso formativo individual del alumno. El tutor tiene un papel transversal en la formación del alumno, perfectamente complementario al del profesor de prácticas dependiente del coordinador de la asignatura.

Finalmente, el hospital debe implicarse -como un todo- en la evaluación objetiva de las competencias adquiridas, con la participación de expertos en educación médica que, junto con los responsables académicos, definirán el mapa de competencias, el cronograma de su adquisición, el ámbito de su aprendizaje, el método para adquirirlas y las pruebas que permitan evaluar su consecución (Tabla III).

\section{Iniciativas en la facultad de medicina}

Las facultades de medicina, conscientes de su responsabilidad, deberían arbitrar propuestas concretas que permitieran y facilitaran los cambios que es preciso introducir, acordes con el papel 
Tabla IV. Estrategias de la facultad de medicina para la adquisición de competencias clínicas.

Definición de competencias

- Para que el alumno conozca lo que tiene que hacer

- Para que el alumno conozca cómo lo debe hacer

- Para que el alumno conozca en qué marco lo debe hacer

Adquisición de competencias

- Aula de habilidades médico-quirúrgica

- Sistema tutorial

- Residencialización' del alumno

- Estancias clínicas. Hospital, centro de salud, laboratorio de investigación

Evaluación de áreas competenciales -objetiva y estructurada (ECOE)-

- Anamnesis

- Exploración física

- Comunicación

- Juicio clínico

- Habilidades

- Conocimientos

- Relaciones profesionales

- Salud/medicina preventiva

- Ético/legal

- Investigación

ECOE: evaluación clínica objetiva estructurada.

que debe desempeñar el hospital universitario en la formación de los futuros médicos.

La Facultad de Medicina de la Universidad Complutense ha planteado tres líneas estratégicas concretas alineadas con una extensa utilización de los recursos hospitalarios para la docencia y que están relacionadas con la definición, adquisición y evaluación de las competencias clínicas, respectivamente (Tabla IV).

La primera se basa en la predefinición de las competencias clínicas que los alumnos deberían alcanzar a su paso por la facultad y el hospital. Ello ha permitido predefinir un total de 1.133 competencias, de las que 460 corresponden a habilidades clínicas y 259 a procedimientos dianósticos y/o terapéuticos. En el proceso de participación multidepartamental tendrá su origen el futuro mapa de competencias definitivo, que habrá de ser la base y el fundamento de la definición de los programas y, en suma, del futuro plan de estudios.

La segunda iniciativa se orienta a la mejora de la adquisición de tales competencias, a través de la instauración de programas o sistemas específicos formativos en los que el alumno puede pasar a ser protagonista de su propia actividad. Se trata del mantenimiento y utilización de aulas de habilidades, y de sistemas asistenciales que faciliten la adquisición de las competencias clínicas, como la implantación del sistema tutorial, la 'residencialización’ del alumno en los servicios medicoquirúrgicos, y las estancias clínicas fuera del período lectivo en los servicios hospitalarios, los centros de atención primaria o los laboratorios de investigación.

Por último, forma parte de la evaluación de los resultados la realización de una prueba de evaluación clínica objetiva estructurada final (en sexto curso), estructurada en dos fases (multiestaciones y prueba escrita), y que permita evaluar de forma ponderada los siguientes campos: anamnesis, exploración clínica, comunicación, habilidades clínicas, conocimientos y juicio clínico, entre otros.

\section{Bibliografía}

1. Barón-Maldonado M. La enseñanza de la medicina. In Villanueva JL, Millán J, Barón M, eds. Estándares para la enseñanza de la medicina. Madrid: Fundación Lilly; 2006. p. 1-11.

2. Martínez-Carretero JM. Los métodos de evaluación de la competencia profesional: la evaluación clínica objetiva estructurada (ECOE). Educación Médica 2005 (Supl 2): S18-22.

3. Millán Núñez-Cortés J. Las competencias clínicas. Educación Médica 2005 (Supl 2): S13-4.

4. Millán Núñez-Cortes J, García-Seoane J, Calvo-Manuel E, Díez-Lobato R, Villanueva-Marcos JL, Nogales-Espert A. La transmisión de los valores médicos: competencias específicas. Educación Médica 2006; 9 (Supl 1): S24-8.

5. Nogales-Espert A. El hospital universitario. En Villanueva JL, Millán J, Barón M, eds. Estándares para la enseñanza de la medicina. Madrid: Fundación Lilly; 2006. p. 11-22.

6. Peinado-Herreros JM. Competencias médicas. Educación Médica 2005 (Supl 2): S4-6. 